\title{
Remarques
}

$1^{0}$ L'acide sulfurique à employer pour la préparation du réactif ci-dessus, doit être d'une très grande pureté.

$2^{\circ}$ Un réactif très ancien (un an par ex.) ne permet plus d'obtenir la réaction caractéristique des alginates.

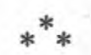

\section{En résumé}

L'étude de ces diverses techniques nous permet de penser qu'un excellent moyen de mettre en évidence la présence d'alginates dans les produits laitiers, consiste :

$1^{0} \mathrm{~A}$ isoler et à purifier l'épaississant en utilisant la technique décrite par Fouassin et reproduite au début de cette note.

$2^{\circ} \mathrm{A}$ identifier les alginates dans l'épaississant ainsi séparé, par le réactif à l'oxyde ferrique en solution sulfurique dont la préparation a été indiquée ci-dessus.

Il va de soi que, sur une autre prise d'essai, la réaction de l'anthrone, également décrite, permet d'obtenir une confirmation qualitative de la présence de cet épaississant de structure glucidique.

\section{BIBLIOGRAPHIE}

[1] Fouassin A. Rev. ferment. ind. alim., 1957, 4, 169.

[2] Racicot et Ferguson. J. Ass. off. agric. chem. 1938, 21, 110.

[3] Schroeder et Racicot. Ind. and. Ingen. chem., 1941, 13, 165.

[4] Bundesen et Martinek. J. milk. food. Techn., 1954, 17, 79 et 105.

\section{LE PROBLèmE du LAIT hyGiÉNIQUE (1)}

\author{
par G. THIEULIN \\ Agrégé des Eeoles Vétérinaires \\ Membre du Conseil Supérieur d'Hygiène publique
}

D'autres que nous, plus qualifiés, ont dit ou vont dire, au cours de ce colloque, la valeur de l'aliment exceptionnel qu'est le lait, mais aussi nos exigenees devant les dangers qu'il peut, s'il est souillé, représenter.

(1) Résumé d'une Conférence prononcée le 20 mai 1959, au Colloque International de Diététique sur le lait et ses dérivés. (Paris, Faculté de Médecine.) 
Aussi, nous faut-il disposer, abondamment, pour tous, d'un lait "hygiénique ", c'est-à-dire procurant ses bienfaits sans nous faire craindre aucun méfait. Problème complexe, d'ordre scientifique, technique et économique, puisque pour répondre à la demande, le lait doit être sain, de composition normale, et d'un prix raisonnable. Or, ce lait est une denrée fragile et, pour approvisionner un centre urbain important, le circuit commercial se révèle semé d'embûches.

Cependant, l'équilibre recherché est à notre portée, sous réserve d'un peu de compréhension et de quelques efforts : tel est le sujet de ce bref exposé.

La composition du lait étant connue, sa valeur alimentaire nettement établie, encore convient-il, qu'aux fins d'une utilisation rationnelle, nous soit livré un produit constant, permettant un régime suffisant, sans excès, toujours équilibré.

Or, la mamelle fabrique un produit variable, surtout en ce qui concerne sa teneur en matière grasse. Dès que l'on procède à un mélange, on tend évidemment, plus ce mélange est grand, à compenser les écarts notés à l'origine, mais on a alors favorisé les contaminations, et, deux heures environ après la traite, les proliférations microbiennes suivent une progression géométrique. N'oublions pas que, dans les cas envisagés, le remise aux consommateurs demande plusieurs heures, souvent une journée entière. Le froid seul, qui ne saurait sans de très onéreuses servitudes être appliqué de façon continue, ne suffirait pas à éviter de graves et irréversibles altérations.

Au surplus, il y a lieu de considérer que, dès son origine, puis par suite des nécessaires manipulations dont il est l'objet, le lait peut contenir des microbes pathogènes.

Et c'est pour ces divers motifs qu'un traitement thermique approprié doit intervenir en vue d'obtenir un lait véritablement hygiénique. Mais il ne saurait s'agir alors d'un mauvais palliatif dont l'incidence heureuse serait simplement de prolonger la vie commerciale d'une denrée polluée.

Trois conditions sont à réunir formellement pour que cette opération, basée sur l'action de la chaleur, nous satisfasse :

$1^{\circ}$ Qu'elle concerne un lait cru de qualité satisfaisante;

$2^{\circ}$ Qu'elle permette de maintenir au maximum les valeurs biologiques initiales de ce produit;

$3^{\circ} \mathrm{Qu}$ 'elle soit complétée par une protection efficace du lait valablement traité.

C'est sur le premier de ces trois points qu'il nous est donné d'insister aujourd'hui. 
D'emblée, il faut nous placer dans la réalité et ne pas nous plaire dans l'utopie. En un mot, nous devons installer l'hygiène dans la vie journalière et tenir compte des faits matériels : la production du lait doit rester économique.

D'autre part, il convient de répéter que le lait cru satisfaisant est un lait pour lequel la garantie réelle sera d'obtention aisée, c'est-à-dire, selon l'heureuse expression de C. GoRINI, un lait de pasteurisation facile, pour lequel une température relativement peu élevée $\left(72^{\circ}\right)$ et une courte durée de chauffage (16 secondes) seront suffisantes en vue de la sécurité bactériologique et sans inconvénient pour le respect de la pleine qualité nutritive.

La situation n'est pas encore aujourd'hui, en général, très favorable, et cela tient à plusieurs causes dont l'examen rapide permet justement de dégager la ligne de conduite à suivre.

Nous sommes ainsi amené à considérer l'état sanitaire des animaux et leurs conditions d'entretien, la récolte du lait et son départ de la ferme, sa destination aussi, et enfin le prix payé au producteur.

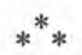

La lutte contre les maladies animales régit au premier plan, la production d'un lait sain; elle est en outre nécessaire à l'amélioration du revenu agricole. Ceci souligne l'importance que revêt la campagne d'éradication de la tuberculose bovine, décidée et organisée par les Pouvoirs publics et qui débuta en 1950 pour prendre, à partir de 1953, une réelle ampleur, en étant coordonnée et conduite sous sa forme actuelle, e'est-à-dire libre et collective, dans le cadre de comités départementaux et de groupements de défense sanitaire.

Sur un total de deux millions d'exploitations et de dix-huit millions de bovins, l'assainissement, mesuré d'après le nombre des exploitations et des animaux passés sous le contrôle des Services Vétérinaires, a progressé, de 1950 à 1956 , de 0,6 à $16 \%$ dans les deux cas; au 31 décembre 1956, ce contrôle concernait environ 320.000 exploitations et trois millions de bovins, 24.000 étables ayant été assainies. Les opérations se sont intensifiées en 1957, année au cours de laquelle 140.000 exploitations, dont $21 \%$ d'infectées, et 1.100 .000 animaux, dont $8,6 \%$ de tuberculeux, ont été pris en charge. Les pourcentages d'infection ont vérifié à nouveau l'exactitude des évaluations faites dès 1954 à la suite de tuberculinations de sondage, et qui avaient permis de considérer qu'en France, environ $25 \%$ des exploitations et $9 \%$ du cheptel bovin étaient infectés. 
Depuis 1954, la progression de l'assainissement s'est poursuivie à la cadence annuelle de 100.000 exploitations et de un million d'animaux passant sous contrôle vétérinaire. Aujourd'hui, environ $25 \%$ 'de l'effectif est contrôlé; il ne faut pas dissimuler que la progression est trop lente et que, pour être bénéfique, l'assainissement devrait être poursuivi à une cadence accélérée. Le chemin parcouru est déjà long, mais il reste encore beaucoup à faire et les menaces de contagion devront maintenir continuellement l'attention.

Sans doute est-il inutile de rappeler la gravité de la tuberculose animale pour l'homme, en raison des infections réciproques. Des études ont établi que le nombre d'infections à bacille bovin chez l'homme était en relation directe avec le degré de tuberculisation du bétail : $64 \%$ de cuti-réactions positives chez des enfants vivant dans des fermes infectées, $11 \%$ dans les exploitations indemnes. La tuberculose humaine, d'origine bovine, est plus fréquente dans les campagnes que dans les villes. Si les contacts avec les bovins tuberculeux peuvent parfois être incriminés, c'est l'ingestion de produits virulents qui représente le mode le plus fréquent d'une contamination qui sévit le plus lourdement pendant le jeune âge, et le lait cru est le grand coupable.

Rappelons, d'après le Dr. G. Cuvirer, que, au Danemark par exemple, il a été établi que le maximum des résultats ayant été atteint dans la lutte contre l'infection inter-humaine, le reliquat de l'endémie était maintenu dans le milieu rural par la tuberculose d'origine bovine. De même, le Professeur et Mme Sorel ont signalé que depuis la disparition de la tuberculose bovine aux Etats-Unis, la tuberculose osseuse ne s'y rencontrait plus ou très exceptionnellement, même dans les Etats du Sud où elle était auparavant fréquente.

La brucellose bovine ne constitue pas, en matière d'hygiène du lait un problème majeur mais, par contre, la brucellose caprine et ovine représente une affection redoutable pour le consommateur de lait de chèvre ou de brebis. Toutefois, en ce qui concerne les grands ruminants, les étables fournissant un lait consommé à l'état cru doivent être l'objet d'une active surveillance.

Les troubles intestinaux et en particulier l'entérite chronique constituent pour la vache laitière des tares indiscutables. Les mammites provoquent la production d'un lait profondément altéré, et nous savons bien que les moyens thérapeutiques modernes, symbolisés par la miraculeuse pénicilline, n'ont pas résolu le problème, et que l'usage inconsidéré des antibiotiques n'empêche pas les réinfections dues à la survivance des agents pathogènes 
dans le milieu extérieur et à l'absence d'une hygiène préventive, seule efficace.

Les conditions de vie de la vache laitière sont étroitement en rapport avec son état sanitaire.

La tuberculose est maladie du logement.

Ceci montre les difficultés rencontrées dans la lutte poursuivie car l'habitat ne peut pas être rapidement amélioré, mais il est crucial de ne pas perdre de vue l'importance qu'il représente.

A ce sujet, nous devons noter les bienfaits d'une modalité nouvelle : la stabulation libre, favorable à la santé du troupeau, à l'hygiène du lait et à l'abaissement des prix de revient. Correctement réalisé et entretenu, puis généralisé, ce nouveau mode d'exploitation semble susceptible, en révolutionnant des habitudes bien ancrées, de marquer un pas décisif dans la voie du progrès.

- L'alimentation des femelles productrices de lait est certes une des bases de l'élevage, mais, en même temps, nous préoccupe. La teneur du lait en vitamines est dépendante à la fois des conditions d'existence et du régime alimentaire. Par ailleurs, le trop large emploi de divers résidus industriels, tels que les tourteaux, les drêches et les pulpes, ou l'usage, même modéré, de ces produits, lorsqu'ils ont été l'objet de fermentations anormales ou qu'ils se trouvent altérés pour d'autres raisons, permet de communiquer au lait des goûts anormaux et peut lui conférer des propriétés toxiques que le chauffage ne supprimera pas, que l'analyse ne pourra percevoir, mais que, très rapidement, la clinique révèle.

La boisson ne saurait être négligée, et des eaux chargées de substances chimiques, souvent résiduaires, ont pu, par leur absorption, donner au lait une nocivité certaine, tout en perturbant les fonctions animales de digestion et de secrétion.

- D'autres motifs d'insalubrité sont encore à éviter : ils se rapportent à la traite, manuelle ou mécanique, au nettoyage du matériel laitier et en particulier aux produits utilisés pour le réaliser, puis encore à l'usage des insecticides et à celui des substances de désinfection, auxquelles on ne prend pas garde avec la rigueur qui pourtant s'impose.

Dans ces différents domaines, l'hygiéniste a d'autant plus la parole que son action protège la santé et, en même temps, sert l'économie.

- Nous n'insisterons pas non plus davantage, sur l'hygiène générale et sur les contaminations banales qui, fréquentes et abondantes, provoquent plus de ravages que les souillures spécifiques et, dès l'étable, altèrent le lait profondément et définitivement. 


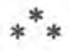

Mais s'il est intéressant, et facile, d'énumérer et même de classer les prescriptions qui seraient à observer pour obtenir au stade initial de la production du lait, le minimum de garanties nécessaires, faute desquelles la sécurité finale demeure aléatoire, quels que soient les correctifs mis en œuvre, il n'est pas moins utile de considérer que trois groupes de facteurs interviennent pour permettre ou empêcher d'atteindre le but fixé. Il ne suffit pas, en effet, de se trouver d'accord sur celui-ci, encore faut-il mettre en œuvre les moyens nécessaires au succès de l'entreprise. Nous voulons parler du prix du lait, des possibilités matérielles de production d'un lait sain, et de la meilleure utilisation de ce lait.

1) Il $y^{*}$ a quelques mois, une note (1) était présentée à la Tribune de l'Académie d'Agriculture, concernant l'étude du prix de revient du lait à la ferme. Bien que s'étant placés dans des conditions exceptionnellement favorables d'exploitation, les A. de la note ont pu écrire que "les prix unitaires payés depuis plusieurs années, non seulement ne laissent aucun bénéfice à l'exploitant mais ne remboursent pas les frais élémentaires ". Nous notons au surplus que les frais élémentaires détaillés, dont il a été question, ne comportaient pas ceux qui seraient fatalement entraînés par des soins particuliers inhérents à une production devenue hygiéniquement satisfaisante.

Citons également que, réunis en octobre 1957, à Rome, les experts du comité européen de la Fédération Internationale des Producteurs agricoles ont pris acte que "dans l'Europe des six puissances associées, et en comprenant en outre la Suisse et la Scandinavie, la France est au dernier rang pour le prix du lait ». Il nous est ainsi apparent qu'en notre pays, le lait est vraiment le parent pauvre parmi les fournitures journalières indispensables à la vie.

Plus néfaste encore, pensons-nous, qu'un prix trop bas, est le prix uniforme. Seule, en effet, la teneur en matière grasse a été jusqu'à présent officiellement estimée : aucun égard n'a été accordé à la salubrité.

2) Nous avons souligné précédemment l'importance de la lutte maintenant entreprise contre les maladies animales, mais nous pouvons répéter que le programme dressé justifierait des crédits beaucoup plus considérables que ceux consentis et que le danger est grand de les voir encore diminuer.

(1) Compte rendus de l'Académie d'Agricult're, 1957, 43, $\mathrm{n}^{\circ} 14,753$. 
Attachons-nous, en passant, à un détail d'ordre très général, concernant l'hygiène élémentaire : il faut environ sept litres d'eau pour concourir à l'obtention d'un litre de lait propre; eneore faut-il que cette eau soit potable, et de préférence qu'elle le soit " naturellement", c'est-à-dire de façon certaine. En outre, il est nécessaire que le producteur dispose d'une source d'énergie facilement maniable, et le courant électrique paraît à ce titre irremplaçable dans la vie moderne. Si cette seconde exigence est en bonne voie d'être partout satisfaite, la première mérite par contre d'être plus activement étudiée.

La question de l'eau à la ferme est du plus haut intérêt.

3) Le lait est un aliment direct, c'est aussi une matière première industrielle. Cette seconde position règle tout et c'est à notre sens une lourde erreur.

En effet, le taux de matière grasse du lait en conditionne, de façon jusqu'alors exclusive, la valeur marchande. Nous aboutissons sur le plan général à une production excédentaire de beurre, ce qui conduit à une exportation réalisée, quand elle peut l'être, sur des bases désastreuses pour l'économie nationale. La valorisation de l'azote du lait serait, par contre, salutaire. Contre la matière grasse de lait trop chère, il est une concurrence facile; contre un lait de consommation qui serait de la qualité désirée, il n'est pas de concurrence possible.

Pour en terminer sur ce point, une remise en ordre s'impose : le lait de consommation directe exige plus de soins et plus de frais que le lait destiné à devenir beurre ou fromage; il doit être pour le producteur, dans l'intérêt du consommateur, non plus une cause de déficit, mais une source de légitime profit.

Hors cela, pas de progrès.

$$
* * *
$$

Nous voulons enfin souligner ici, parmi quelques autres, la réalisation d'un établissement modèle, la Laiterie Coopérative du Mans, dont la politique hardie et intelligente, basée naturellement sur un prix de vente parfaitement justifié (1), aboutit à la livraison d'un excellent lait de consommation : il s'agit évidemment d'un lait pasteurisé, traité rationellement selon les normes partout reconnues comme les meilleures, et qui peut être obtenu parce que le lait cru utilisé est propre et peu microbien, qu'il

(1) Contrat pour la livraison de lait aux Services des Etats.Unis, stationnés en France. 
provient d'étables saines, correctement aménagées, et qu'il est, dès la traite, refroidi.

Il nous semble inutile d'insister en ce lieu, sur les qualités d'une telle fourniture, couramment commercialisée à l'étranger, à la satisfaction générale.

Nous possédons donc, et ee sera notre conclusion, toutes les informations nécessaires et nous connaissons les moyens efficaces, sanitaires, techniques et économiques, pour répandre à profusion le lait hygiénique, si bienfaisant.

Nous avons sous les yeux, en France, un exemple salutaire et qui se révèle durable.

La raison et le courage devraient, sans plus de retard, faire prendre, sur le plan national, des décisions impatiemment attendues et hautement motivées, en faveur de l'hygiène publique et de l'économie nationale.

\title{
SUPPLEMENT TECHNIQUE
}

\section{PRÉPARATION ET PROPRIÉTÉS DE DIFFÉRENTS COMPOSÉS DÉRIVÉS DE CORPS GRAS ET DE L'ACIDE LACTIQUE}

\author{
par G. GÉNIN \\ Ingénieur E.P.C.
}

Dans le but de préparer une série de nouveaux composés susceptibles de trouver des applications comme constituants de différents produits alimentaires, les laboratoires de la société américaine Proctor and Gamble Co [1] ont préparé une série de glycérides de l'acide lactique de structure connue. En général, la méthode de synthèse de ces produits a été inspiré de celle décrite précédemment par GoLdBLATT et ses collaborateurs [2].

L'acide lactique a été introduit dans la molécule de glycéride sous la forme d'un dérivé de l'acide O-benzyllactique. Le groupe benzyle était ensuite éliminé par hydrogénolyse, soit pour obtenir soit le produit désiré, soit pour rendre le groupe hydroxyle de l'acide lactique libre en vue de réactions ultérieures. Les propriétés de ces produits ont été comparées à celles d'autres dérivés des corps gras et on a également examiné l'activité interfacielle de ces produits, qui contiennent des groupes hydroxyles, dans les systèmes graisse et eau. 